\title{
COVARIANT AND CONTRAVARIANT APPROACHES TO TOPOLOGY
}

\author{
JERZY DYDAK \\ Department of Mathematics, \\ University of Tennessee. \\ Knoxville, Tennessee 37996 \\ (Received April 22, 1997 and in revised form June 12, 1997)
}

\begin{abstract}
This paper is an exposition of results contained in [2]. The purpose of [2] is to present a way of viewing of basic topology which unifies quite a few results and concepts previously seemed not related (quotient maps, product topology, subspace topology, separation axioms, topologies on function spaces, dimension, metrizability). The basic idea is that in order to investigate an unknown space $X$. one either maps known spaces to $X$ or maps $X$ to known spaces.
\end{abstract}

KEYWORDS AND PHRASES: function spaces, quotient spaces, local compactness, compactopen topology

1991 AMS SUBJECT CLASSIFICATION CODES: 54B15, 54B17, 54C35, 54C20

\section{INTRODUCTION}

In [1] the author presented certain results of basic topology from the point of view of Extension Theory. In [2] we broaden the approach of [1]. Namely, extension theory can be viewed as part of the contravariant approach, and it makes sense to ponder its dual, the covariant approach.

Suppose we have a class of known spaces $K$, and we are faced with an unknown space $X$. We may choose one of the following strategies:

1. (Covariant approach) $X$ will be investigated by considering maps $f: K \rightarrow X$ from known spaces to $X$.

2. (Contravariant approach) $X$ will be investigated by considering maps $f: X \rightarrow K$ from $X$ to known spaces. 
The covariant approach is widely used in the clasical homotopy theory and leads to homotopy/homology groups (see [11]). The contravariant approach is the mainstay of shape theory (see [10]), cohomological dimension theory (see [12]), and leads to cohomology/cohomotopy groups. However, in basic topology the prevalent approach is that of intrinsic definitions/theorems in terms of open sets/covers.

The purpose of this paper is to translate the intrinsic approach of basic topology into covariant/contravariant approaches in an effort to unify various concepts which seemed unrelated up to now (quotient maps, product topology, subspace topology, separation axioms, dimension, metrizability). We believe that it brings better understanding and better results. Also, it allows to integrate basic topology with category theory at an earlier stage. Let us explain this statement in the case of function spaces.

One of the fundamental concepts in category theory is that of adjoint functors (see [6]), and in algebra one creates the tensor product as a left adjoint to the Hom functor. Thus, one proves the Adjoint Associativity Theorem of algebra (see Theorem 5.10 in [5] on p.214): $\operatorname{Hom}_{R}\left(M \otimes_{R} N, P\right) \sim \operatorname{Hom}_{R}\left(M, \operatorname{Hom}_{R}(N, P)\right)$. In the category of sets a left adjoint to the Hom functor is the cartesian product functor. In the category of topological spaces the problem is reversed: the cartesian product functor is well-understood and one has difficulty defining the Hom functor. The difficulty lies in choosing a natural topology on the function space $Y^{X}$ of all maps from $X$ to $Y$. Through various trials and errors topologists created the so-called compact-open topology which works very well on the category of compactly generated spaces (also known as $k$-spaces or Kelley spaces). In [2], a more natural approach was chosen: Since the bijection $\operatorname{adj}_{Y}: \operatorname{Hom}_{\text {Sets }}\left(X, \operatorname{Hom}_{S e t s}(Y, Z)\right) \rightarrow \operatorname{Hom}_{S e t s}(X \times Y, Z)$ is given by $\operatorname{adj}_{Y}(f)(x, y)=$ $f(x)(y)$, let us declare $f: X \rightarrow \operatorname{Hom}_{T o p}(Y, Z)$ to be continuous if $\operatorname{adj}_{Y}(f)$ is continuous. This leads to the basic covariant topology on function spaces. Thus, the goal of [2] is, from the beginning, to discuss topologies on function spaces so that the resulting Hom functor is a right adjoint to the cartesian product functor via the function $\operatorname{adj}_{Y}$, i.e. we are aiming at adjY : $\operatorname{Hom}_{\text {Top }}\left(X, \operatorname{Hom}_{T o p}(Y, Z)\right) \rightarrow \operatorname{Hom}_{\text {Top }}(X \times Y, Z)$ to be a natural homeomorphism. In the terminology of [6], adj $j_{Y}$ is the adjugant equivalence (or adjugant) and.one is led to the front adjunction (the resulting natural transformation from the identity functor to the composition Hom $\circ x$ ) and the rear adjunction (the resulting natural transformation from the composition $\times \circ$ Hom to the identity functor). The front adjunction corresponds to the function $\beta=a d j^{Y}(i d)$ : $X \rightarrow(X \times Y)^{Y}$ given by $\beta(x)(y)=(x, y)$ and is clearly continuous by the definition of the basic covariant topology. The rear adjunction eval : $X \times Y^{X} \rightarrow Y$ is the well-known evaluation function: $\operatorname{eval}(x, f)=f(x)$. Thus, the question of Hom being right adjoint to the cartesian product reduces to the question of continuity of the evaluation function. It turns out that it is connected to the Whitehead-Michael [9] characterization of locally compact spaces as those spaces $Z$ for which $f \times i d_{Z}$ is a quotient map if $f$ is a quotient map. This leads quickly to establishing that, on the category of locally compact spaces, the functor Hom defined as the function space equipped with the basic covariant topology is a right adjoint to the cartesian product functor. Since most questions regarding compactly generated spaces can be reduced to the locally compact case, one can apply the functor $k: T o p \rightarrow k T o p$ from the topological spaces 
to the $k$-spaces and obtain Hom on the category $k T o p$ which is right adjoint to the product functor (in $k T o p$ ). Obviously, this particular functor Hom must be $Y^{X}$ with the compact-open topology (by the uniqueness of adjoint functors) but the approach of [2] is much better integrated with the category theory and, therefore, allows natural and functorial proofs.

There is a dual approach to the one described above: given a way of assigning topologies on function spaces we try to find topologies on the cartesian product so that one obtains a left adjoint functor to $G(Z)=M a p_{T o p}(Y, Z)$ if $Y$ is fixed. There are two cases of interest; $T o p=P C$ being the point convergence topology or $T o p=C O$ being the compact-open topology. It is shown in [2] that one can introduce the PC-product $X \times_{P C} Y$ and the CO-product $X \times_{C O} Y$ so that if $T o p \in\{P C, C O\}$, then one has a natural equivalence

$$
a d j^{Y}: M a p_{T o p}\left(X \times_{T o p} Y, Z\right) \rightarrow M a p_{T o p}\left(X, M a p_{T o p}(Y, Z)\right)
$$

for all spaces $X, Y, Z$. One may say that those products are topological analogs of the tensor product in algebra. It turns out that the PC-product is commutative and the CO-product is not commutative.

In the next two sections we discuss the basic results of [2] without proofs. The detailed proofs can be found in [2] as well as an extended discussion of various topologies on the function spaces.

\section{COVARIANT AND CONTRAVARIANT TOPOLOGIES}

1.1. Definition. The topology $\mathcal{T}$ on $X$ is called the covariant topology induced by the class of functions $\mathcal{F}=\left\{f: X_{f} \rightarrow X\right\}$ if each $X_{f}$ is a topological space and $\mathcal{T}$ is the largest of all topologies on $X$ under which all $f \in \mathcal{F}$ are continuous.

Notice that the covariant topology exists and consists of all sets $U$ such that $f^{-1}(U)$ is open for each $f \in \mathcal{F}$. This leads to:

1.2. Example. A surjective function $f: X \rightarrow Y$ is a quotient map iff the topology on $Y$ is the covariant topology induced by the single function $\{f\}$.

1.3. Example. Given a set $\left\{X_{s}\right\}_{s \in S}$ of topological spaces, the classical topology on the disjoint union $\coprod_{s \in S} X_{s}$ is the covariant topology induced by inclusions $i_{t}: X_{t} \rightarrow \bigsqcup_{s \in S} X_{s}, t \in S$.

1.4. Example. Given a simplicial complex $K$, the weak topology $|K|_{w}$ is the covariant topology induced by all inclusions $i_{\Delta}:|\Delta|_{m} \rightarrow|K|$, where $\Delta$ is a simplex in $K$ and $|\Delta|_{m}$ is $|\Delta|$ equipped with the standard metric topology.

One of the basic classes of topological spaces are Fréchet spaces (see Section 1.6 of [3]):

1.5. Proposition. $X$ is a Fréchet space iff its topology is the covariant topology induced by a family of functions from $\{0\} \cup\{1 / n \mid n \geq 1\} \subset \mathbf{R}$ to $X$.

The dual to the notion if the covariant topology is the contravariant topology:

1.6. Definition. The topology $\mathcal{T}$ on $X$ is called the contravariant topology induced by the class of functions $\mathcal{F}=\left\{f: X \rightarrow X_{f}\right\}$ if each $X_{f}$ is a topological space and $\mathcal{T}$ is the smallest of all topologies on $X$ under which all $f \in \mathcal{F}$ are continuous. 
Notice that the contravariant topology exists and its sub-basis consists of all sets $f^{-1}(U)$, where $U$ is open in $X_{f}$ for some $f \in \mathcal{F}$.

1.7. Example. If $A$ is a subset of a topological space $X$, then the subspace topology on $A$ is the contravariant topology induced by the inclusion $i_{A}: A \rightarrow X$.

1.8. Example. An injective map $f: X \rightarrow Y$ is a homeomorphic embedding iff the topology on $X$ is the contravariant topology induced by $\{f\}$.

1.9. Example. The product topology on the cartesian product $\prod_{s \in S} X_{s}$ is the contravariant topology induced by projections $\left\{\pi_{t}: \prod_{s \in S} X_{s} \rightarrow X_{t}\right\}_{t \in S}$.

The basic property of covariant topologies is:

1.10. Proposition. Suppose the topology of $X$ is the covariant topology induced by a class of functions $\left\{f_{\imath}: X_{\imath} \rightarrow X\right\}_{i \in J}$. Then, a function $g: X \rightarrow Y$ is continuous iff $g \circ f_{2}$ is continuous for all $\imath \in J$.

The basic result regarding covariant topologies is:

1.11. Theorem. Suppose the topology on $X$ is the covariant topology induced by a class of maps $\left\{f_{s}: X_{s} \rightarrow X\right\}_{s \in S}$ so that $X=\bigcup_{s \in S} f_{s}\left(X_{s}\right)$. If $Z$ is locally compact, then the covariant topology induced by $\left\{f_{s} \times i d_{Z}: X_{s} \times Z \rightarrow X \times Z\right\}_{s \in S}$ is the product topology on $X \times Z$, where each $X_{s} \times Z$ is equipped with the product topology.

The basic property of contravariant topologies is:

1.12. Proposition. Suppose the topology of $X$ is the contravariant topology induced by a class of functions $\left\{f_{2}: X \rightarrow X_{2}\right\}_{i \in J}$. Then, a function $g: Y \rightarrow X$ is continuous iff $f_{\imath} \circ g$ is continuous for each $i \in J$.

\section{BASIC CONCEPTS IN TOPOLOGY FROM COVARIANT/CONTRAVARIANT POINTS OF VIEW}

Let us assume that the following spaces are well-understood:

1. Anti-discrete spaces (spaces with the smallest topology possible),

2. Discrete spaces (spaces with the largest topology possible), including the integers $\mathbf{Z}$ and natural numbers $\mathbf{N}$,

3. $S^{0}$ (the 0-dimensional sphere or the simplest discrete space which is not anti-discrete),

4. The unit interval $I$ with the standard topology,

5. The real numbers $\mathbf{R}$ with the standard topology. $\mathbf{Q} \subset \mathbf{R}$ are rationals.

It is well known that connected spaces $X$ are precisely those, so that all maps $f: X \rightarrow$ $S^{0}$ are constant. Thus, connectedness is a contravariant property. On the other hand, path connectedness is a covariant property as $X$ is path connected iff any map $f: S^{0} \rightarrow X$ extends over $I$. Let us analyze basic concepts of topology from those two points of view.

\subsection{Proposition.}

1. $X$ is $T_{0}$ iff any map $f: A \rightarrow X$ from an anti-discrete space $A$ to $X$ is constant.

2. $X$ is $T_{1}$ iff any non-constant map $f: S^{0} \rightarrow X$ is a homeomorphic embedding. 
3. Suppose $X$ is $T_{1}$. Then, $X$ is $T_{2}$ (Hausdorff) iff $S^{0}$ is an absolute neighborhood extensor of $X$ with respert to finite subspaces.

4. Suppose $X$ is $T_{0}$. Then, $X$ is $T_{3 \frac{1}{2}}$ (Tychonoff) iff the topology of $X$ is the contravariant topology induced by a family of functions $\left\{f_{s}: X \rightarrow I\right\}_{s} \in S$.

5. Suppose $X$ is $T_{1}$. Then, $X$ is $T_{4}$ (normal) iff $S^{0}$ is an absolute neighborhood extensor of $X$.

6. Suppose $X$ is $T_{1}$. Then, $X$ is collectionwise normal iff all discrete spaces $D$ are absolute neighborhood extensors of $X$.

The purest contravariant approximation of compactness is pseudo-compactness (see 3.10 of $[3])$ :

$X$ is called pseudo-compact if any map $f: X \rightarrow \mathbf{R}$ from $X$ to reals is bounded.

The following result summarizes well-known characterizations of Hausdorff compact spaces in terms which are contravariant in spirit:

2.2. Theorem. Suppose $X$ is Hausdorff. The following conditions are equivalent:

1. $X$ is compact,

2. $X$ is regular and any map $f: X \rightarrow Y$ from $X$ to a Hausdorff space is closed,

3. $X$ is regular and $f(X)$ is closed in $Y$ for any map $f$ from $X$ to a Hausdorff space $Y$,

4. $X$ is regular and $f$ is a homeomorphic embedding for any injective map $f$ from $X$ to a Hausdorff space $Y$.

The following well-known result of Tamano (see Theorem 5.1.38 in [3]) can be interpreted that paracompactness is a contravariant property:

2.3. Theorem (Tamano). $X \in T_{2}$ is paracompact iff $X \times C$ is normal for all compact Hausdorff spaces $C$.

The following metrizability criterion proved by the author in [1] means that metrizability is a contravariant property:

2.4. Theorem. $X \in T_{0}$ is metrizable iff the topology of $X$ is the contravariant topology induced by a set of maps $\left\{f_{s}: X \rightarrow I\right\}_{s \in S}$ such that

$$
\sum_{s \in S} f_{s}=1 .
$$

Theorem 2.4 was improved in [1] as follows:

2.5. Theorem. $X \in T_{0}$ is metrizable iff there is a set of maps $\left\{f_{s}: X \rightarrow I\right\}_{s \in S}$ such that

$$
\sum_{s \in S} f_{s}=1
$$

and $\left\{f_{s}^{-1}(0,1]\right\}_{s \in S}$ is a basis of $X$.

Theorem 2.5 implies the well-known metrizability criteria, Kuratowski-Wojdysławski Theorem, and Arens-Eells Theorem (see [1]).

Completness in the sense of Čech is a covariant property (see Theorem 3.9.1 of [3]):

2.6. Proposition. Suppose $X$ is a Tychonoff space. Then, $X$ is complete in the sense of Cech iff any map $f: A \rightarrow X$ from a subset $A$ of a Tychonoff space $Y$ extends over a $G_{\delta}$ subset of $Y$.

Being a $k$-space is a covariant property (see Theorem 3.3 .18 of $[3]$ ): 
2.7. Proposition. $X$ is a $k$-space (also known as compactly generated) iff the topology of $X$ is the covariant topology induced by a functions of maps

$$
\left\{f_{s}: C_{s} \rightarrow X\right\}_{s \in S}
$$

from locally compact spaces to $X$.

The following well-known result underscores the importance of Theorem 1.11 (see Theorem 3.3.27 of [3]):

2.8. Corollary. If $X$ is a $k$-space and $Y$ is locally compact, then $X \times Y$ is a $k$-space.

Being of covering dimension $n$ is a contravariant property (see [7]):

2.9. Theorem (Hurewicz-Wallman). $\operatorname{dim}(X) \leq n$ iff $S^{n} \in A E(X)$.

Theorem 2.9 explains why the covering dimension is the most widely used of all theories of dimension.

In [1] the author proved the following generalization of Tietze-Urysohn Theorem and Urysohn Lemma:

2.10. Theorem. Suppose $Y=\{$ point $\}$ is a Hausdorff space. Then, the following conditions are equivalent:

1. $\operatorname{Cone}(Y) \in A E(X)$,

2. $(\operatorname{Cone}(Y), Y) \in A E(X)$,

3. $Y \in A N E(X)$.

Traditionally, the $C o n e(Y)$ of $Y$ is understood as the quotient space $Y \times I / Y \times\{0\}$. That would mean that the topology of the cone is introduced in a covariant manner. If one wants to map spaces to the cone, then as seen in Proposition 1.13, it is better to introduce a topology on the cone in a contravariant manner. Notice that there are two natural functions: the projection $p_{I}: C o n e(X) \rightarrow I$ and the projection $p_{X}: C o n e(X)-p t \rightarrow X$. These two functions define a contravariant topology on the cone $\operatorname{Con} e(Y)$ which is equivalent to the one introduced in [1]. Thus, for general spaces $Y$, one has two kinds of cones: the covariant cone and the contravariant cone. In the case of a metric space $Y$, the covariant cone may not be metrizable but the contravariant cone is metrizable (use 2.4). Theorem 2.10 deals with contravariant cones.

\section{REFERENCES}

[1] J. Dydak, Extension theory: The interface between set-theoretzc and algebrazc topology, Topology and its Appl. 20 (1996), 1-34.

[2] J. Dydak, Covariant and contravariant points of vrew in topology wrth applzcatzons to function spaces. preprint (1997).

[3] R. Engelking, General Topology, Berlin, 1989.

[4] S.T. Hu, Theory of retracts, Wayne State University Press. 1965.

[5] T.W.Hungerford, Algebra, Springer-Verlag, New York, 1974.

[6] P.J.Hilton and U.Stammbach, A Course in Homological Algebra, Springer-Verlag, 1971.

[7] W. Hurewicz and H. Wallman, Dimenszon Theory, Princeton University Press, 1941.

[8] S.Mac Lane, Categories for the workng mathematician, Springer-Verlag, 1972.

[9] E. Michael, Local compactness and Carteszan products of quotient maps and $k$-spaces, Ann. Inst. Fourier 18 (1968), 281-286.

[10] S. Mardesicí and J. Segal, Shape theory, North-Holland Publ.Co., Amsterdam, 1982.

[11] E. Spanier, Algebrazc topology, McGraw-Hill, New York, 1966.

[12] J.J.Walsh, Dimenszon, cohomologrcal dimension, and cell-like mappings, Lecture Notes in Math. 870, 1981, pp. 105-118. 


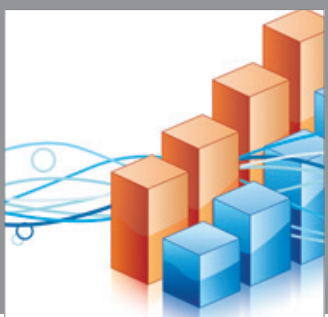

Advances in

Operations Research

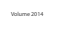

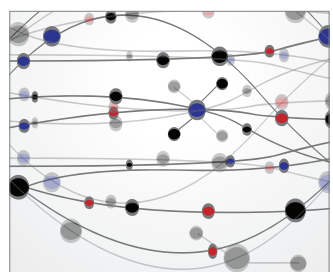

\section{The Scientific} World Journal
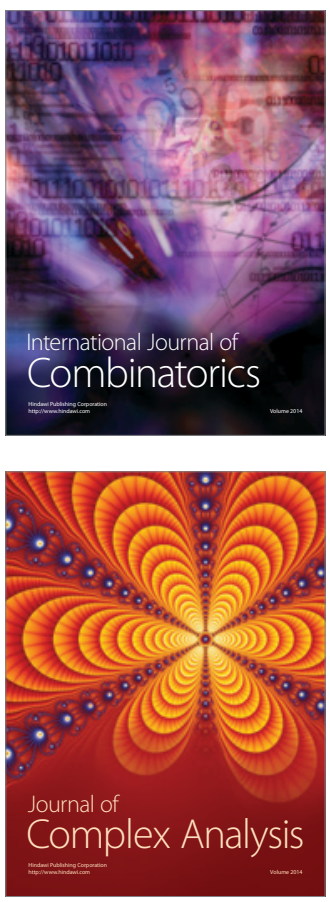

International Journal of

Mathematics and

Mathematical

Sciences
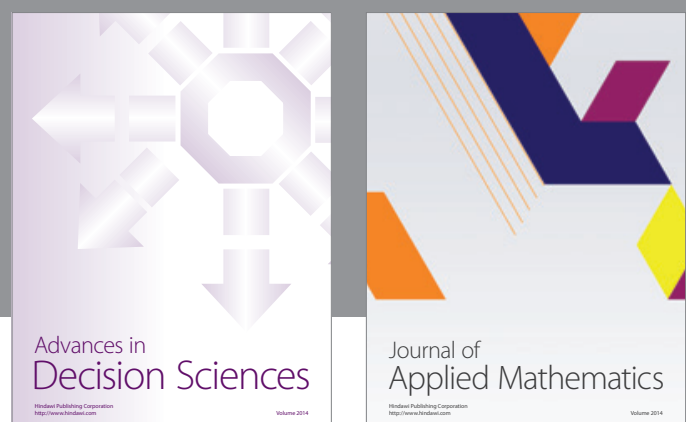

Journal of

Applied Mathematics
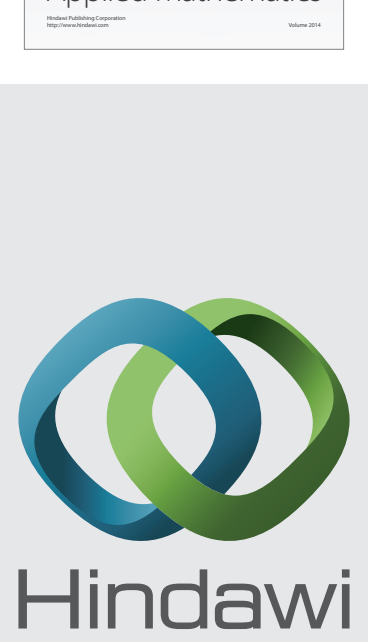

Submit your manuscripts at http://www.hindawi.com
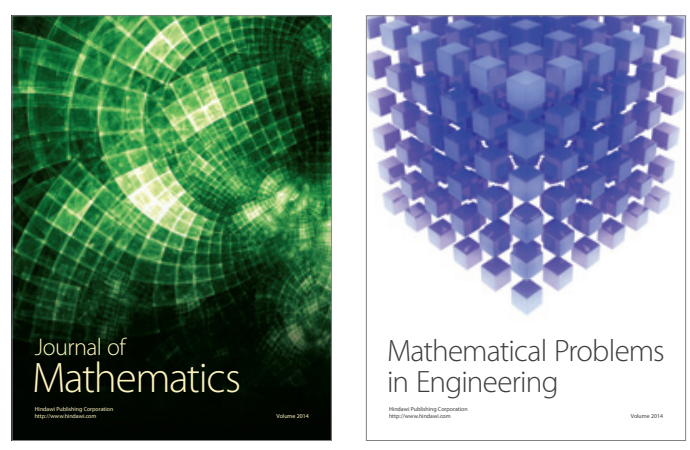

Mathematical Problems in Engineering
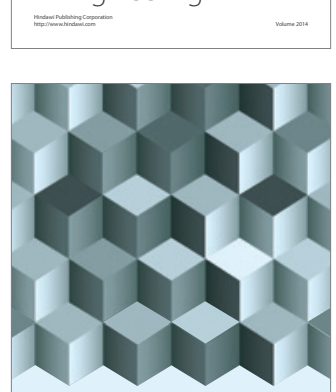

Journal of

Function Spaces
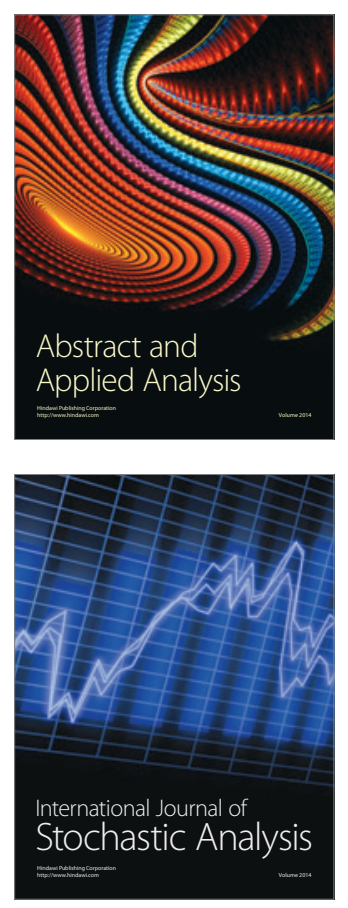

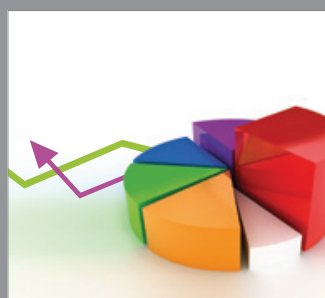

ournal of

Probability and Statistics

Promensencen
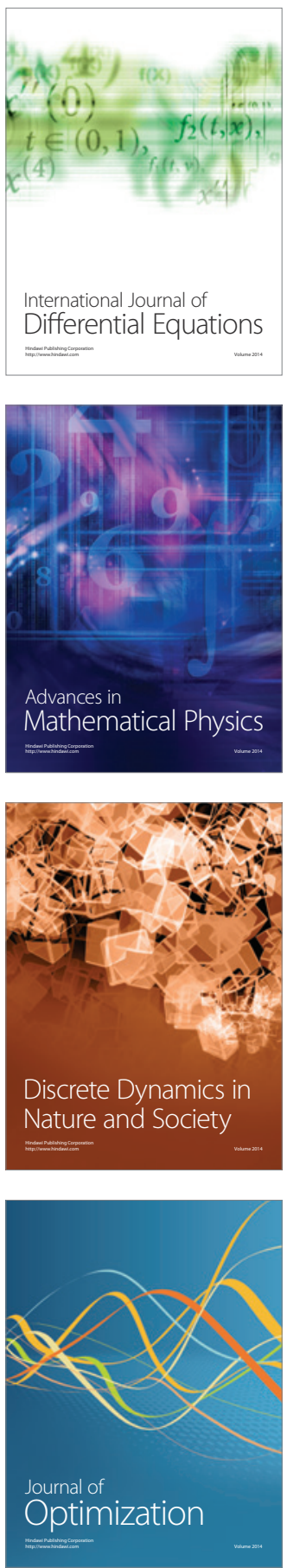